\title{
Integrated Navigation Method and Realization Based on Monocular and IMU for Quadrotor
}

\author{
CunXiao MIAO ${ }^{a}$ and WeiMing WEN ${ }^{\text {b, }}{ }^{*}$ MingHua LU ${ }^{c}$ \\ School of Mechanical Engineering, University of Science and Technology Beijing, Beijing 10083, \\ China \\ amiaocx@ustb.edu.cn, b849302437@qq.com, 'c13263406073@163.com
}

Keywords: Monocular, IMU, Quadrotor, Navigation.

\begin{abstract}
This paper realizes the localization method base on the SVO (Semi-Direct Visual Odometry) and IMU (Inertial Measurement Unit) without GPS. According to this novel visual estimation method, the position and orientation are estimated by the MSF (Multi Sensor Fusion) with EKF (Extended Kalman Filter). In order to consider the actual application, the accuracy and the repeatability are evaluated and analyzed. For this purpose, two types of experiments are performed, in which the accuracy and the repeatability of our navigation platform are described and analyzed.
\end{abstract}

\section{Introduction}

At present, the positioning and navigation of the quadrotor is a key problem to expand the application range of the UAV. Accurate navigation information of quadrotor is the foundation of flight control, flight path planning, autonomous complete tasks and other complex tasks.

In this paper, an integrated navigation based on monocular vision and IMU is studied. After reading a great deal of paper, $\mathrm{SVO}^{[1]}$ monocular vision algorithms and MSF ${ }^{[2]-[4]}$ algorithm which based on EKF method is introduced to an integrated navigation method. According to the integrated navigation method we build a quadrotor navigation platform, the platform consist of hardware and software components. Software is based on Ubuntu which is integrated ROS robot framework ${ }^{[5,6]}$ and hardware consist of a camera from IDS and flight controller and NUC computer.

Finally, after the quadrotor system is set up and two types of experiment of the aircraft will be carried out and the navigation data of the platform will be analyzed.

\section{SVO algorithm}

SVO algorithm, one of the novelest vision algorithms, integrates the advantages of direct method and feature-based method. So the efficiency of the SVO algorithm is much faster than one of them. SVO algorithm is mainly composed of motion estimation thread and 3D point map reconstruction thread, and the motion estimation thread is the core of the SVO algorithm.

The motion estimation thread is divided into three steps. At first, all the information of the last frame, including the image information of the last frame and the rigid body transformation information $T$ of the last frame, and the image information collected by the current frame will be aligned in the sparse model-based image alignment step. In this step, from the image pixels, which will be projected to the corresponding 3D point that is named back-projecting 2D point $\mathrm{u}$, and related current 2D point we can get the intensity residual $\delta I$ as Eq. 1.

$$
\delta I(T, \mathrm{u})=I_{k}\left(\pi\left(T \cdot \pi^{-1}\left(u, d_{u}\right)\right)\right)-I_{k-1}(u) \quad \forall u \in \bar{R}
$$

Here $\bar{R}$ is the image region for which the depth $d_{u}$ is known. After calculating the minimum of intensity residual with least square method, the rigid body transformation $T_{k, k-1}$ which we will get as Eq. 2 can be used as input in the next steps. 


$$
\mathrm{T}_{k, k-1}=\arg \min _{\mathrm{T}_{k, k-1}} \frac{1}{2} \sum_{i \in \bar{R}}\left\|\delta I\left(\mathrm{~T}_{k, k-1}, u_{i}\right)\right\|^{2}
$$

In the second step, by comparing the intensity of the 3D feature points from mapping thread which project to the current frame image to the intensity of current frame, the intensity residual based on feature alignment will be optimize by as Eq. 3 where the $A_{i}$ is affine warping.

$$
u_{i}^{\prime}=\arg \min \frac{1}{2}\left\|I_{k}\left(u_{i}^{\prime}\right)-A_{i} \cdot I_{r}\left(u_{i}\right)\right\|^{2}, \forall i
$$

Finally the rigid body transformation will be optimized by geometric characteristics. Then the rigid body transformation will be optimized by calculating the minimum of the geometric distance between the pixel of the current frame and back-projecting 2D point as Eq. 4.

$$
\mathrm{T}_{k, w}=\arg \min _{\mathrm{T}_{k, w}} \frac{1}{2} \sum_{i}\left\|u_{i}-\pi\left(\mathrm{T}_{k, w},_{w} p_{i}\right)\right\|^{2}
$$

Finally the information from the motion estimation thread including the intensity of the image and rigid body transformation will be used to create the 3D points in the mapping thread. So after the all information are input to the mapping thread, it will determine whether it is a key frame or not. If it is a key frame, the depth filter will be initialed. Else, the depth of the filter will be updated. When the uncertainty of the depth is less than the depth filter, the point will be inserted to the 3D points.

\section{MSF algorithm}

MSF algorithm can fuse the rigid body transformation from both SVO algorithm and IMU, which is used to generate the optimal estimator. Firstly, we can built the system equations for the quadrotor system, which included the differential equation and sensor error and initial value information $b_{\omega}, b_{a}$, $n_{b_{\omega}}, n_{b_{a}}, \lambda, p_{i}^{s}, q_{i}^{s}$ of the aircraft system as Eq. 5. The differential equation consist of the relationship between the velocity $v_{\omega}^{i}$ and the motion $p_{\omega}^{i}$, the acceleration $a_{m}, b_{a}, n_{a}$ and the velocity $v_{\omega}^{i}$, and the relationship of angular velocity $\omega_{m}, b_{\omega}, n_{\omega}$.

$$
\left\{\begin{array}{l}
\dot{p}_{\omega}^{i}=v_{\omega}^{i} \\
\dot{v}_{\omega}^{i}=C_{\left(q_{\omega}^{i}\right)}^{T}\left(a_{m}-b_{a}-n_{a}\right)-g \\
\dot{q}_{\omega}^{i}=\frac{1}{2} \Omega\left(\omega_{m}-b_{\omega}-n_{\omega}\right) q_{\omega}^{i} \\
\dot{b}_{\omega}=n_{b_{\omega}} \quad \dot{b}_{a}=n_{b_{a}} \quad \dot{\lambda}=0 \quad \dot{p}_{i}^{s}=0 \quad \dot{q}_{i}^{s}=0
\end{array}\right.
$$

According the system equation of quadrotor, we can get the states of the system as Eq. 6, which is used to get the optimal estimator of the rigid transformation by MSF algorithm based on EKF.

$$
\mathbf{x}=\left\{\begin{array}{llllllll}
p_{\omega}^{i T} & v_{\omega}^{i T} & q_{\omega}^{i T} & b_{\omega}^{T} & b_{a}^{T} & \lambda & p_{i}^{s} & p_{i}^{s}
\end{array}\right\}
$$

MSF algorithm is divided into two steps, the first step is the state prediction, and the second step is the state update step. In the prediction step, after setting up the initial values, the prediction states $\hat{\mathbf{x}}_{t \mid t-1}$ and prediction error $\mathbf{P}_{t \mid t-1}$ will be calculated according to the system equation as Eq.7.

$$
\left\{\begin{array}{l}
\hat{\mathbf{x}}_{t \mid t-1}=\mathbf{F}_{t} \hat{\mathbf{x}}_{t-1 \mid t-1}+\mathbf{B}_{t} \mathbf{u}_{t} \\
\mathbf{P}_{t \mid t-1}=\mathbf{F}_{t} \mathbf{P}_{t-1 \mid t-1} \mathbf{F}_{t}^{\mathbf{T}}+\mathbf{Q}_{t}
\end{array}\right.
$$


Here $\mathbf{F}_{t}$ is state-transition function and $\mathbf{B}_{t} \mathbf{u}_{t}$ is control signal and $\mathbf{Q}_{t}$ is used to represented the covariance of the process noise. In the second step, according to the prediction error from the first step the kalman gain $\mathbf{K}_{t}$ will be determined. And the visual measurements and the IMU measurements $\mathbf{z}_{t}$ will be fused with the kalman gain as Eq. 8.

$$
\left\{\begin{array}{l}
\mathbf{K}_{t}=\mathbf{P}_{t \mid t-1} \mathbf{H}_{t}^{\mathrm{T}}\left(\mathbf{H}_{t} \mathbf{P}_{t \mid t-1} \mathbf{H}_{t}^{\mathrm{T}}+\mathbf{R}_{t}\right)^{-1} \\
\hat{\mathbf{x}}_{t \mid t}=\mathbf{F}_{t} \hat{\mathbf{x}}_{t \mid t-1}+\mathbf{K}_{t}\left(\mathbf{z}_{t}-\mathbf{H}_{t} \hat{\mathbf{x}}_{t \mid t-1}\right) \\
\mathbf{P}_{t \mid t}=\mathbf{P}_{t \mid t-1}-\mathbf{K}_{t} \mathbf{H}_{t} \mathbf{P}_{t \mid t-1}
\end{array}\right.
$$

Here $\mathbf{H}_{t}$ is sensor function and $\mathbf{R}_{t}$ is used to represented the covariance of the measurement noise. On one hand the optimal state prediction $\hat{\mathbf{x}}_{t \mid t}$ and the update error $\mathbf{P}_{t \mid t}$ will be used to provide the navigation information, on the other hand they will be used as input in the next state prediction step. So, we can use MSF algorithm to get the optimal state estimation of quadrotor which will be used to provide the navigation information by integrate all the optimal state estimation.

\section{Navigation platform and experiments}

A navigation platform is built according to the SVO algorithm and MSF algorithm. The navigation platform consist of the camera form IDS and flight control module and Intel NUC onboard computer. After the navigation platform setting up, two type of experiments is carried on. The first kind of experiment is about landing at the speed less than $0.1 \mathrm{~m} / \mathrm{s}$.
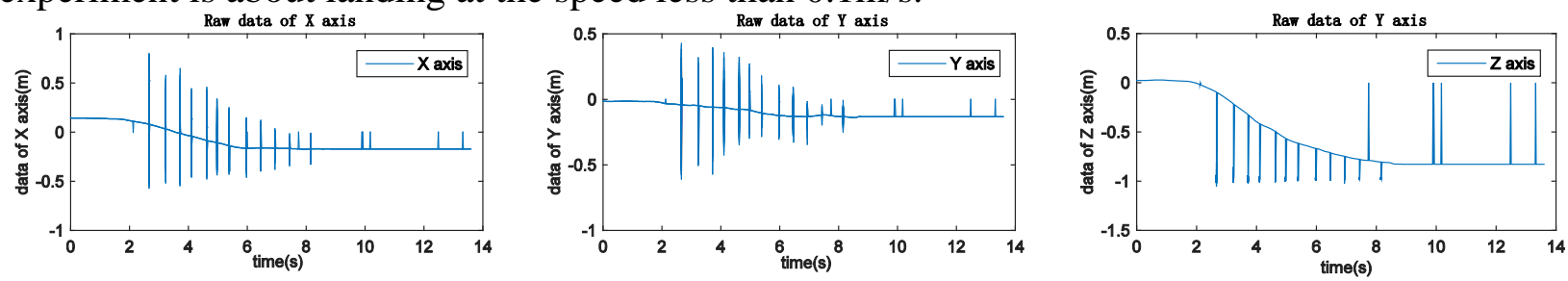

Fig. 1 Raw data
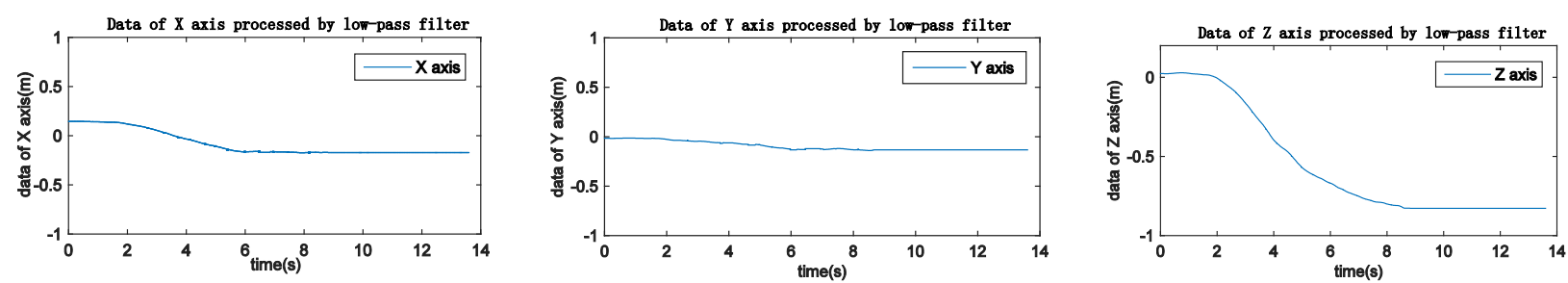

Fig 2 Processed data

According to the data of landing experiment, it is very easier to find there are a lot of outlier point of the moving as Fig. 1 . So we randomly choose the datas of $x$ axis, which are used to analyzed the outlier point by draw the picture as Fig. 3 according sample number.

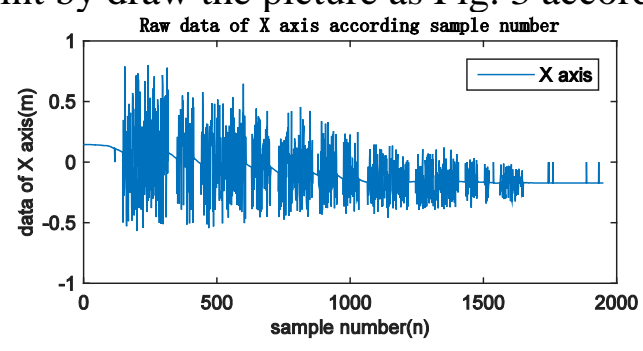

Fig. 3 Raw data according sample number

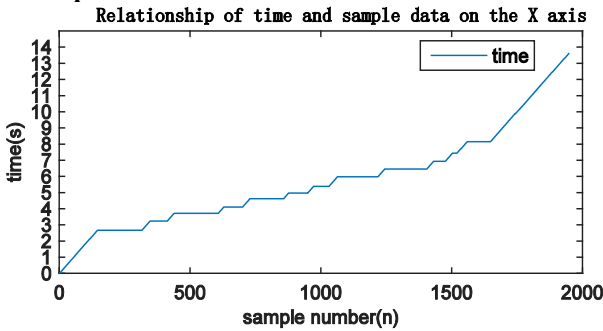

Fig. 4 Relationship of time and sample time

Regarding to the the related picture in the Fig. 1, the more outlier points can be founded in the Fig. 3. And we can easier to know that the outlier points are closely related to time as it show in the Fig. 4. 
And the Fig. 4 reveal that the key of the outlier points is that, in some period of time, the navigation platform has process a lot of sampling point, Which mean the process of the navigation method is so fast that we could see the navigation platform is flying as Fig. 2 in the really world. Finally we can see the trajectory of navigation platform in the 3D world as Fig. 5.

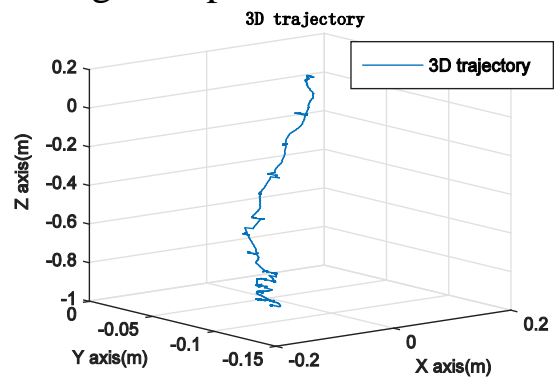

Fig. 5 3D trajectory

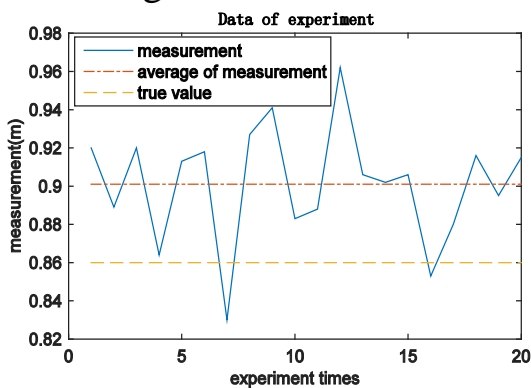

Fig. 6 Data of 20 times experiment

In order to analyze the accuracy and the repeatability of our navigation platform, we repeat the landing experiment 20 times and the data are shown in the Fig. 6, where we can find the average of measurement is $0.901 \mathrm{~m}$, the average error between the true value is $0.041 \mathrm{~m}$, the average relative error is $4.81 \%$, and the biggest error is $0.11 \mathrm{~m}$. And the standard deviation of measurement data is 0.03 , which means the repeatability of our navigation platform is good enough to use in the interior.

The second experiment is moving our navigation platform according to a square trajectory or a trajectory like number 8 as Fig. 7. The drift of our platform is really small which means that our platform can go back to the point where it take off as Fig. 7.

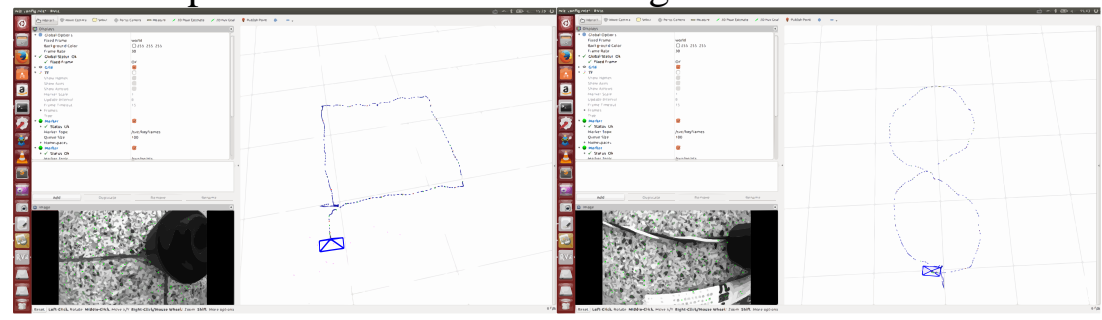

Fig. 7 trajectory of square and number 8

\section{Conclusions}

The integrated navigation method consist of SVO algorithm and MSF algorithm, which provide information for indoor or others without GPS environment. The navigation platform is built by us which is used to realize the navigation method and some experiments is used to prove the efficiency and the accuracy of the navigation system.

\section{References}

[1] Christian Forster, Matia Pizzoli. Robotics and Automation (ICRA), 2014 IEEE International Conference on, pp. 15-22, May 31 2014-June 72014

[2] S Weiss, MW Achtelik. Robotics and Automation (ICRA), 2012 IEEE International Conference on, pp. 31-38, May 2012

[3] S Weiss, R Siewart. Robotics and Automation (ICRA), 2011 IEEE International Comference on, pp. 4531-4537, May 2011

[4] Stephan M. Weiss. "Vision Based Navigation for Micro Helicopters” Ph.D. dissertation, Swiss Federal Institute of Technology Zurich, Switzerland, 2012

[5] Information on http://chriative.com/blog/projects/

[6] Information on http://pixhawk.org/dev/ros/visual_estimation 